\title{
Men Ejaculate Larger Volumes of Semen, More Motile Sperm, and More Quickly when Exposed to Images of Novel Women
}

\author{
Paul N. Joseph ${ }^{1,3} \cdot$ Rakesh K. Sharma $^{2} \cdot$ Ashok Agarwal $^{2} \cdot$ Laura K. Sirot $^{1}$
}

Published online: 13 June 2015

(C) Springer International Publishing 2015

\begin{abstract}
Males in many species differentially allocate sperm and seminal fluid depending on certain social variables, including perceived sperm competition and female reproductive status. In some species, males reduce their investment in sperm quantity or quality upon repeated matings with the same female and increase such investment when mated to a novel female. We tested for effects of stimulus habituation and novelty on ejaculated semen parameters in humans. We analyzed ejaculates produced through masturbation with stimulation from sexually explicit films. When males were exposed successively to the same female six times, we saw no change in ejaculate parameters between the first and sixth exposures to the same female. However, ejaculate volume and total motile sperm count significantly increased when males were exposed to a novel female. Time to ejaculation also decreased significantly upon exposure to a novel female. Thus, our results suggest that human males ejaculate more quickly and invest more in ejaculates with novel females.
\end{abstract}

Keywords Strategic ejaculation · Differential allocation · Sperm competition $\cdot$ Human

Electronic supplementary material The online version of this article (doi:10.1007/s40806-015-0022-8) contains supplementary material, which is available to authorized users.

Laura K. Sirot

1sirot@wooster.edu

1 Department of Biology, The College of Wooster, Wooster, OH, USA

2 Center for Reproductive Medicine, Cleveland Clinic, Mail Code X-11, 10681 Carnegie Avenue, Cleveland, OH 10681, USA

3 Present address: Entomology and Nematology Department, University of Florida, Gainesville, FL, USA
Competition between males for fertilization of a female's eggs can result in selection on a variety of phenotypic traits, including morphology, behavior, physiology, and ejaculate composition. In the case of ejaculate composition, selection may favor differential allocation of sperm and other ejaculate components depending on intrinsic (e.g., the male's own condition) or extrinsic factors (e.g., presence of competitors or condition of the females; Perry et al. 2013). Differential allocation of sperm based on exposure to competitor males and on female qualities occurs in a wide range of species (reviewed in Wedell et al. 2002; Kelly and Jennions 2011; also see Evans et al. 2003; Pizzari et al. 2003; delBarco-Trillo and Ferkin 2006; Thomas and Simmons 2007; Lüpold et al. 2011). Males of some species have also been found to differentially allocate seminal fluid proteins (reviewed in Perry et al. 2013). To date, there has been very little research examining differential allocation of ejaculate components in humans; however, work thus far suggests that it does occur (reviewed in Leivers and Simmons 2014; see also Baker and Bellis 1993; Kilgallon and Simmons 2005; Leivers et al. 2014). For example, men who viewed sexually explicit images suggesting sperm competition (i.e., two men with one woman) produced a greater proportion of motile sperm in their ejaculates than men who viewed sexually explicit images suggesting no competition (i.e., three women; Kilgallon and Simmons 2005). In the current study, we tested whether ejaculate parameters change in response to the familiarity or novelty of the female stimulus used to induce sexual arousal.

In many species, including humans, individuals have the opportunity to mate with either a familiar partner (one with whom they have already mated) or with a novel partner (one with whom they have not previously mated). Some species show a pattern of decreased investment in matings with familiar partners and increased investment in matings with novel partners (Beach and Jordan 1956; Dewsbury 1981; Pizzari 
et al. 2003; Jordan and Brooks 2010). Such variable investment can include time, energy, and resources allocated toward courtship, the actual mating process, and/or ejaculate components in the case of males. It is predicted that sexual selection would favor males who invest more in ejaculates transferred to novel females for two reasons: (i) Males may have already fertilized the egg(s) of (or have their sperm stored by) females with whom they have already mated (Jordan and Brooks 2010); and (ii) novel females may be more likely to have mated recently with another male resulting in increased likelihood of sperm competition. Consistent with this prediction, when male jungle fowl, Gallus gallus, mated with a novel female after mating repeatedly to a familiar female, they transferred significantly more sperm than they had in the previous mating with the familiar female (Pizzari et al. 2003). These results support the hypothesis that males differentially allocate sperm depending on their past mating history with a particular female.

The objective of the current study was to test if ejaculate parameters of human males change in response to familiarity or novelty of a female stimulus. We compared several traits between ejaculates produced after six repeated exposures to sexually explicit films depicting the same actress and actor and then after subsequent exposure to a similar film with a new actress but the same actor. The ejaculate traits we compared were ejaculate volume, percent sperm motility, sperm concentration, total sperm count, and total motile sperm count, all of which we predicted to decrease with repeated exposure to the same female and increase upon exposure to a novel female. We also measured time to ejaculation for which we predicted an increase with repeated exposure to the same female and a decrease upon exposure to a novel female.

\section{Materials and Methods}

\section{Test Subjects}

The experiment involved the participation of 23 self-identified heterosexual men between the ages of 18 and 23 years old. Each subject provided seven ejaculate samples over the course of 15 days. The recruitment and procedures involving collection of semen samples were all in accordance with the College of Wooster's Human Subjects Research Committee guidelines. Although we knew the identities of the participants, which remained confidential, all other information, including personal health issues and semen parameters, was anonymous. Prior to beginning the experiment, each subject was assessed for healthiness and history of sexual dysfunction, as defined by the World Health Organization (2010), using a questionnaire. Men were only included in the study if they self-reported to have no history of sexual dysfunction, no conditions affecting testicular health, and no sexually transmitted infections and were not taking any medications, were not regular smokers of tobacco or any other substance, and did not chew tobacco regularly. Prior to beginning the experiment, each participant refrained from alcohol consumption and sexual activity for $48 \mathrm{~h}$. Before each ejaculate collection, participants completed a survey self-reporting the number of alcoholic drinks they had consumed and whether they had ejaculated since the last collection. If they reported having more than four alcoholic drinks or they ejaculated between collections, the participants' data were not included in the study. One participant chose to leave the study and his data were excluded. Another participant's data were excluded because he ejaculated between collections, thus reducing our sample size to 21 .

\section{Collection Procedure}

We used segments of sexually explicit films involving one actor and one actress as the stimuli for the experiment. Each participant viewed the films in the same private room, at approximately the same time of day every 48 to $72 \mathrm{~h}$. For each participant, all sessions were scheduled in advance at the beginning of his participation period. Each film segment consisted of a 3-min section from a longer 20 -min film. This 3-min segment was played on repeat for as long as necessary to achieve ejaculation. Of the seven film segments each participant viewed, the first six comprised the habituation period. Each of these six film segments featured the same man and woman but differed in respect to the sexual acts performed. The seventh film segment comprised the novel female treatment. This film segment featured the same man, but with a different woman who differed distinctly from the first woman in facial and body features, hair color, and tattoos. Across the study, four different 20-min films were used. Each man was assigned to a particular pair of 20-min films, and the segments he viewed were extracted from these films. The specific films used as the habituation film and the novel stimulus film for each participant were randomly assigned. The specific segments from those films that the participant viewed on a particular collection day were also randomized.

Each participant was left alone in a private room to watch the provided film and masturbate into a wide-mouth sterile collection cup. He was instructed to record what time he began watching the film, what time he ejaculated, and whether all of the ejaculate was deposited in the collection cup. If some ejaculate failed to be deposited into the cup, the data from the ejaculate were not used for calculations of volume-based measures (see below).

\section{Semen Assays}

We performed all semen assays blind to the identity and the treatment group (familiar or novel stimulus) of the participant 
and within an hour after ejaculation. Ejaculate volume was measured to the nearest $0.1 \mathrm{~mL}$ using a $15 \mathrm{~mL}$ graduated conical tube. Once in the tube, the sample was gently vortexed and placed into $1.5 \mathrm{~mL}$ Eppendorf tubes. The samples were then allowed to liquefy for $20 \mathrm{~min}$. After vortexing one of the $1.5 \mathrm{~mL}$ tubes again to make the sample homogenous, $5 \mu \mathrm{L}$ of the sample were loaded on a Microcell counting chamber (Vitrolife, San Diego, CA) to record sperm concentration and motility. Using a phase contrast microscope at $20 \times$ objective and an eyepiece video camera, we filmed five arbitrarily chosen fields of view close to the center of the counting chamber slide for a minute each.

To determine sperm concentration and percent motility, we counted total sperm number and total immotile sperm. We used Adobe Illustrator and Adobe Photoshop to place a $10 \times$ 10 grid on top of the sperm videos. For each of the five fields of view of each sample that were videotaped, we counted the total number of sperm and the number of immotile sperm within 1-10 rows (10-100 squares) of the grid. The number of rows counted depended on how concentrated the sample was with sperm. We only counted one to two rows for each field of view for highly concentrated samples (average of 1632 sperm cells per row). If samples were moderately concentrated, five rows were counted (average of eight sperm cells per row). If there were fewer than five sperm cells in a row, all ten rows were counted. The number of rows counted for each of the five fields of view for a given sample remained consistent.

Sperm concentration was calculated by multiplying the average total sperm counts for the five fields of view by the row factor $(1$ row $=10 ; 2$ rows $=5 ; 5$ rows $=2 ; 10$ rows $=1)$ and the magnification factor of the microscope $(\mathrm{MF}=20)$ and then dividing by 100 , the total number of grid cells. Total sperm count was calculated as the product of sperm concentration and ejaculate volume. Sperm motility for each of the five fields of view was calculated as the total number of motile sperm divided by the total number of sperm, multiplied by 100. The average of these five values was used as the percent sperm motility measure for the ejaculate sample. Total motile sperm was calculated as the product of total sperm count and the percent sperm motility.

\section{Statistical Analyses}

We tested for differences between the initial trial and the habituated trial (trials 1 and 6) and between the habituated trial and the novel stimulus trial (trials 6 and 7) for all of the measured variables using either one-tailed paired $t$ tests (after natural log transformation for time to ejaculation) or one-tailed Wilcoxon signed-rank tests (total motile sperm and concentration) in SPSS version 22. For all ejaculate measures (sperm parameters and volume), we predicted a decrease with habituation and an increase upon exposure to a novel stimulus. For time until ejaculation, we predicted an increase with habituation and a decrease upon exposure to a novel stimulus. Data from some participants were excluded from the volume, total motile sperm, and total sperm comparisons because some of their ejaculate missed the collection container (four men excluded in the trial 1 vs. 6 comparison, two men excluded in the trial 6 vs. 7 comparison). Data from one additional participant was excluded from all analyses due to extremely low sperm concentration $(<1$ million sperm per $\mathrm{mL})$. Additionally, we compared each ejaculate parameter across trials 2 through 6 to determine whether there were any changes across the habituation period. To do so, we used a repeated-measures ANOVA for the parameters that were normally distributed and had homogeneity of variances across these trials (volume and natural $\log$ transformed time to ejaculation) and pairwise Wilcoxon tests (trial 2 vs. trial 3, trial 3 vs. trial 4 , etc.) for all of the other parameters (reported in Supplemental Tables 1 and 2).

\section{Results}

\section{Variation in Ejaculate Parameters}

We found tremendous intra- and inter-subject variation in all ejaculate parameters. Time to ejaculation ranged between 4 and $21 \mathrm{~min}$. Ejaculate volume ranged between 0.4 and $6.8 \mathrm{~mL}$. Percent motility ranged between 32.6 and $81.1 \%$. Sperm concentration ranged between $4.5 \times 10^{6}$ and $132.9 \times$ $10^{6}$ sperm per $\mathrm{mL}$. Total sperm ranged between $10.3 \times 10^{6}$ and $279.7 \times 10^{6}$. Total motile sperm ranged between $4.2 \times 10^{6}$ and $200.6 \times 10^{6}$

\section{Habituation}

We found no evidence for a habituation effect. In response to repeated exposure with the initial female and male stimulus, none of the measured semen parameters differed significantly between trials 1 and 6 (Table 1). When we compared across the habituation trials 2 through 6 , we found no consistent patterns of change for any of the ejaculate parameters (Supplemental Tables 1 and 2).

\section{Effect of Novel Stimulus}

We found evidence for a novel female effect on ejaculate traits. In response to the novel female stimulus (with the original male stimulus), ejaculate volume and total number of motile sperm in the ejaculate increased significantly (between trials 6 and 7; volume: $t=1.84 ; \mathrm{df}=17 ; p=0.041$; total motile sperm: $Z=1.76 ; N=18 ; p=0.039)$. Time to ejaculation also decreased in response to the novel female stimulus $(t=-1.78$; $\mathrm{df}=19 ; p=0.045)$. None of the other measured semen 
Table 1 Mean differences $( \pm \mathrm{SE})$ between sperm parameters upon repeated exposure to the same female and male actors (trial 6 trial 1 values) and upon exposure to a novel female with the original male actor (trial 7-trial 6 values)

\begin{tabular}{|c|c|c|c|}
\hline $\begin{array}{l}\text { Comparison } \\
\text { Parameter }\end{array}$ & $\begin{array}{l}\text { Habituation } \\
\text { Trial 6-trial } 1\end{array}$ & $\begin{array}{l}\text { Novel stimulus } \\
\text { Trial 7-trial } 6\end{array}$ & Statistical results \\
\hline Ln time to ejaculation (min) & $0.09 \pm 0.07(20)$ & $-0.14 \pm 0.08 *(20)$ & $\begin{array}{l}1=6: t=1.23 ; \mathrm{df}=19 ; p=0.117 \\
6>7: t=-1.78 ; \mathrm{df}=19 ; p=0.045\end{array}$ \\
\hline Ejaculate volume (mL) & $-0.28 \pm 0.38(16)$ & $0.19 \pm 0.11 *(18)$ & $\begin{array}{l}1=6: t=-0.72 ; \mathrm{df}=15 ; p=0.24 \\
6<7: t=1.84 ; \mathrm{df}=17 ; p=0.041\end{array}$ \\
\hline Sperm concentration $\left(\times 10^{6} / \mathrm{mL}\right)$ & $4.05 \pm 4.94(20)$ & $-0.31 \pm 3.94(20)$ & $\begin{array}{l}1=6: Z=0.97 ; N=20 ; p=0.322 \\
6=7: Z=-4.83 ; N=20 ; p=0.629\end{array}$ \\
\hline Percent sperm motility & $3.24 \pm 2.76(20)$ & $0.55 \pm 1.35(20)$ & $\begin{array}{l}1=6: t=1.17 ; \mathrm{df}=19 ; p=0.255 \\
6=7: t=0.411 ; \mathrm{df}=19 ; p=0.686\end{array}$ \\
\hline Total sperm $\left(\times 10^{6}\right)$ & $5.31 \pm 18.68(16)$ & $5.42 \pm 6.52(18)$ & $\begin{array}{l}1=6: t=0.284 ; \mathrm{df}=15 ; p=0.780 \\
6=7: t=0.830 ; \mathrm{df}=17 ; p=0.418\end{array}$ \\
\hline Total motile sperm $\left(\times 10^{6}\right)$ & $7.05 \pm 11.93(16)$ & $4.89 \pm 4.63 *(18)$ & $\begin{array}{l}1=6: Z=0.10 ; N=16 ; p=0.918 \\
6<7: Z=1.76 ; N=18 ; p=0.039\end{array}$ \\
\hline
\end{tabular}

Sample sizes are in parentheses. One-tailed $p$ values reported for differences in predicted direction. Two-tailed $p$ values reported otherwise. ${ }^{*} p<0.05$ parameters changed significantly in response to the novel female stimulus (Table 1).

\section{Discussion}

Our findings are the first to demonstrate that men's ejaculate behavior and composition change in response to a novel female stimulus. When men were exposed to a novel female stimulus after repeated exposure to a different female stimulus, time to ejaculation decreased whereas ejaculate volume and total motile sperm in the ejaculate increased. Our results have important implications for understanding selective pressures on male reproductive patterns, the plasticity of ejaculate allocation, and diagnosis and treatment in the context of male fertility.

Our findings of decreased time to ejaculation and increased ejaculate volume and number of motile sperm with the novel female stimulus suggest selective pressures in our evolutionary past for greater investment in and more rapid transfer of ejaculates in matings with females other than a male's social partner (i.e., extra-pair copulations). An increase in the total number of motile sperm may result in higher likelihood of fertilization and greater ability to compete with other male's sperm, whereas a decrease in the time to ejaculation may decrease the likelihood of an extra-pair copulation being detected. Our results complement the findings of Koukounas and Over (2000) that penile circumference and self-reported arousal also increased upon exposure to a novel female stimulus after habituation to a different female stimulus. In addition to evidence for effects of novel female stimuli, other human studies have demonstrated ejaculate adjustment in response to perceived sperm competition risk (Baker and Bellis 1993; Kilgallon and Simmons 2005). Together, these results show that, although we still do not have a complete understanding of how strong selection from sperm competition has been in the evolutionary past of humans (Simmons et al. 2004; Larmuseau et al. 2013; Leivers and Simmons 2014; Shackelford et al. in press), male physiology and behavior respond in such a way consistent with a history of sperm competition and extra-pair mating opportunities.

Although we found an effect of a novel female stimulus on some ejaculate parameters, we found no effects of repeated exposure to the same female and male stimuli. Previous studies have found evidence for sexual habituation in men. Male arousal, measured by penile tumescence, decreased upon repeated exposure to the same sexually explicit stimulus over shorter (i.e., less than $60 \mathrm{~min}$; O'Donohue and Geer 1985; Plaud et al. 1997; Koukounas and Over 2000; Dawson et al. 2013) and longer time periods (i.e., 3-6 weeks; O’Donohue and Plaud 1994; Plaud et al. 1997). In contrast, we did not find significant changes in any of the ejaculate parameters we measured between a man's first and sixth exposure to stimulus material involving the same actor and actress over an 1114 day period. The only trends in our data that were consistent with a habituation effect were a decrease in ejaculate volume $(t=-0.72 ; \mathrm{df}=15 ; p=0.24)$ and an increase in time until ejaculation $(t=1.23 ; \mathrm{df}=19 ; p=0.12)$. Interestingly, these were two of the three parameters that significantly changed with the novel female exposure. It is possible that more profound changes in these parameters would occur in the habituation phase with increased number of exposures to the familiar female.

The contrasting results of our study and those of previous studies could also be because our study differed from previous studies in two fundamental ways. First, we tested for habituation to the actress and/or actor in the film clip by exposing males repeatedly to clips containing the same people but 
different randomly selected scenes. In contrast, previous studies which found a habituation effect on male sexual arousal used the same exact stimulus material repeatedly and were, thus, testing for habituation to any aspect of the material. It is possible that exposure to different sexual acts in the habituation phase of our study added variation to the ejaculate parameters which, in turn, decreased our ability to discern any significant habituation effects. In the future, it will be important to distinguish between habituation to a particular stimulus material, which includes both the same people and the same scene, and habituation to a particular person or people. Second, our study tested for the effect of habituation on a set of ejaculate parameters, whereas previous studies tested for effects on arousal. The association between sperm parameters and arousal has not been well-studied, although there is some evidence that they are positively associated (Zavos 1985; Zavos and Goodpasture 1989; van Roijen et al. 1996). In the case of habituation studies, tests of effects on arousal response do not allow for the participants to ejaculate during the session. Therefore, decreases in male arousal upon repeated exposure to a particular female stimulus could be a physiological response to perceived thwarted mating attempts. It may be costly for males to become repeatedly aroused without ejaculation. In a natural setting, arousal may result in males pursuing a mate which can be both energetically costly and risky. If the costs of repeated arousal to the same female are not outweighed by benefits of successful copulation (e.g., insemination, strengthening of pair bond), arousal in this scenario may be selected against. Finally, it is possible that ejaculate parameters not measured in our study followed patterns consistent with habituation.

Future studies should evaluate whether decreased investment in ejaculate parameters with repeated exposure to the same female stimulus occurs using different experimental conditions. Six sexual encounters over the course of a 2week period, as used in our study, would have a low likelihood of conception (Zinaman et al. 1996; Thoma et al. 2013). As a result, habituation effects may be seen after more prolonged exposure. Further, we predict that male investment would be more likely to decrease with increased exposure to the same female under conditions in which male ejaculate components were limited. This condition could be induced by decreasing the time between ejaculate collections. If these predictions are not supported, it may be that effects of repeated exposure to the same stimulus affects ejaculate composition differently in species with pregnancy and short durations of sperm retention (e.g., many mammals; Holt and Lloyd 2010) than in species that lay eggs and have long-term sperm storage (e.g., many arthropods, amphibians, fish, birds, and reptiles; Holt and Lloyd 2010). It could be, for example, that males in the former type of species only decrease ejaculate investment after pregnancy is detected.
In addition to contributing insights to the growing literature surrounding human evolutionary biology and sexual selection, the results of this study are of relevance to fertility medicine. Specifically, this study suggests that male infertility could be under-diagnosed since ejaculate samples used to test for infertility and subfertility are often collected with the use of images depicting women other than the man's partner (Yamamoto et al. 2000). However, this effect could be counteracted by the higher fertility of ejaculates produced during coitus than produced during masturbation (e.g., Zavos 1985; Sofikitis and Miyagawa 1993). Our results suggest that it will be important for future studies comparing the fertility of ejaculates produced under different conditions and studies of male fertility diagnoses to consider the effects of stimulus by novel females and sexual scenarios. Considerations of the effect of novel stimuli may result in different conclusions for such studies and diagnoses. Promoting techniques that more closely match normal sexual scenarios used by patients during conception attempts may improve the accuracy of male infertility diagnoses, whereas use of novel stimuli may improve the outcome of assisted reproductive techniques.

In conclusion, in our study, men produced higher quality ejaculates when exposed to novel, rather than familiar, women. Additionally, men ejaculated more quickly when viewing a new woman after being exposed to the same woman repeatedly. These data suggest that males preferentially invest more into novel sexual situations involving unfamiliar women. Further research could be conducted to elucidate the proximate mechanisms influencing differential ejaculate allocation in humans. Additionally, further investigation of the composition of ejaculates, including seminal fluid protein analysis, could unveil other ejaculate components that change with novel stimuli. These future studies could assist medical professionals in devising improved strategies for male infertility diagnosis and for assisted reproductive technology by providing insights into the causes of semen parameter variation in humans.

Acknowledgments We are grateful to the College of Wooster and the Center for Reproductive Medicine at Cleveland Clinic Foundation for their support of this project. We thank T. Pizzari and S. Wigby for helpful discussions of the design of this project and J. Gros-Louis, M. Pham, D. Sasson, S. Wigby, and one anonymous reviewer for insightful feedback on earlier versions of this manuscript.

\section{References}

Baker, R. R., \& Bellis, M. A. (1993). Human sperm competition: ejaculate adjustment by males and the function of masturbation. Animal Behaviour, 46, 861-885.

Beach, F. A., \& Jordan, L. (1956). Sexual exhaustion and recovery in the male rat. The Quarterly Journal of Experimental Psychology, 8, $121-133$. 
Dawson, S. J., Suschinsky, K. D., \& Lalumiere, M. L. (2013). Habituation of sexual responses in men and women: a test of the preparation hypothesis of women's genital responses. Journal of Sexual Medicine, 10(4), 990-1000.

delBarco-Trillo, J., \& Ferkin, M. H. (2006). Male meadow voles respond differently to risk and intensity of sperm competition. Behavioral Ecology, 17(4), 581-585.

Dewsbury, D. A. (1981). Effects of novelty of copulatory behavior: the Coolidge effect and related phenomena. Psychological Bulletin, 89(3), 464-482.

Evans, J. P., Pierotti, M., \& Pilastro, A. (2003). Male mating behavior and ejaculate expenditure under sperm competition risk in the eastern mosquitofish. Behavioral Ecology, 14(2), 268-273.

Holt, W. V., \& Lloyd, R. E. (2010). Sperm storage in the vertebrate female reproductive tract: how does it work so well? Theriogenology, 73(6), 713-722.

Jordan, L. A., \& Brooks, R. C. (2010). The lifetime costs of increased male reproductive effort: courtship, copulation and the Coolidge effect. Journal of Evolutionary Biology, 23(11), 2403-2409.

Kelly, C. D., \& Jennions, M. D. (2011). Sexual selection and sperm quantity: meta-analyses of strategic ejaculation. Biological Reviews of the Cambridge Philosophical Society, 86(4), 863-884.

Kilgallon, S. J., \& Simmons, L. W. (2005). Image content influences men's semen quality. Biology Letters, 1, 253-255.

Koukounas, E., \& Over, R. (2000). Changes in the magnitude of the eyeblink startle response during habituation of sexual arousal. Behaviour Research and Therapy, 38(6), 573-584.

Larmuseau, M. H. D., Vanoverbeke, J., Van Geystelen, A., Defraene, G., Vanderheyden, N., Matthys, K., Wenseleers, T., \& Decorte, R. (2013). Low historical rates of cuckoldry in a Western European human population traced by Y-chromosome and genealogical data. Proceedings of the Royal Society of London B, 280, 1-8.

Leivers, S., \& Simmons, L. W. (2014). Human sperm competition: playing a defensive strategy. Advances in the Study of Behavior, $46,1-44$.

Leivers, S., Rhodes, G., \& Simmons, L. W. (2014). Context-dependent relationship between a composite measure of men's mate value and ejaculate quality. Behavioral Ecology, 25(5), 1115-1122.

Lüpold, S., Manier, M. K., Ala-Honkola, O., Belote, J. M., \& Pitnick, S. (2011). Male Drosophila melanogaster adjust ejaculate size based on female mating status, fecundity, and age. Behavioral Ecololgy, 22, 184-191.

O'Donohue, W. T., \& Geer, J. E. (1985). The habituation of sexual arousal. Archives of Sexual Behavior, 14(3), 233-246.

O'Donohue, W. T., \& Plaud, J. J. (1994). The conditioning of human sexual arousal. Archives of Sexual Behavior, 23(3), 321-344.

Perry, J. C., Sirot, L., \& Wigby, S. (2013). The seminal symphony: how to compose an ejaculate. Trends in Ecology \& Evolution, 28(7), 414 422.
Pizzari, T., Cornwallis, C. K., Løvlie, H., Jakobsson, S., \& Birkhead, T. R. (2003). Sophisticated sperm allocation in male fowl. Nature, 426, $70-74$.

Plaud, J. J., Gaither, G. A., Henderson, S. A., \& Devitt, M. K. (1997). The long-term habituation of sexual arousal in human males: a crossover design. The Psychological Record, 47(3), 385-398.

Shackelford, T. K., Goetz, A. T., LaMunyon, C. W., Pham, M. N., \& Pound, N. (in press). Human sperm competition. In D. M. Buss (Ed.), The handbook of evolutionary psycology (2nd ed.). New York: Wiley.

Simmons, L. W., Firman, R. C., Rhodes, G., \& Peters, M. (2004). Human sperm competition: testis size, sperm production and rates of extrapair copulations. Animal Behaviour, 68, 297-302.

Sofikitis, N. V., \& Miyagawa, I. (1993). Endocrinologic, biophysical, and biochemical parameters of semen collected via masturbation versus sexual intercourse. Journal of Andrology, 14(5), 366-373.

Thoma, M. E., McLain, A. C., Louis, J. F., King, R. B., Trumble, A. C., Sundaram, R., \& Buck Louis, G. M. (2013). Prevalence of infertility in the United States as estimated by the current duration approach and a traditional constructed approach. Fertility and Sterility, 99(5), 1324-1331.

Thomas, M. L., \& Simmons, L. W. (2007). Male crickets adjust the viability of their sperm in response to female mating status. The American Naturalist, 170(2), 190-195.

Van Roijen, J. H., Slob, A. K., Gianotten, W. L., Dohle, G. R., van der Zon, A. T. M., Vreeburg, J. T. M., \& Weber, R. F. A. (1996). Sexual arousal and the quality of semen produced by masturbation. Human Reproduction, 11(1), 147-151.

Wedell, N., Gage, M. J. G., \& Parker, G. A. (2002). Sperm competition, male prudence and sperm-limited females. Trends in Ecology \& Evolution, 17(7), 313-320.

World Health Organization. (2010). WHO laboratory manual for the examination and processing of human semen (5th ed.). Geneva: World Health Organization.

Yamamoto, Y., Sofikitis, N., Mio, Y., \& Miyagawa, I. (2000). Influence of sexual stimulation on sperm parameters in semen samples collected via masturbation from normozoospermic men or cryptozoospermic men participating in an assisted reproduction programme. Andrologia, 32, 131-138.

Zavos, P. M. (1985). Seminal parameters of ejaculates collected from oligospermic and normospermic patients via masturbation and at intercourse with the use of a Silastic seminal fluid collection device. Fertility and Sterility, 44(4), 517-520.

Zavos, P. M., \& Goodpasture, J. C. (1989). Clinical improvements of specific seminal deficiencies via intercourse with a seminal collection device versus masturbation. Fertility and Sterility, 51(1), 190193.

Zinaman, M. J., Clegg, E. D., Brown, C. C., O’Connor, J., \& Selevan, S. G. (1996). Estimates of human fertility and pregnancy loss. Fertility and Sterility, 65(3), 503-509. 